\title{
The Higgs boson mass in minimal technicolor models
}

\author{
A. Doff* and A. A. Natale ${ }^{\dagger}$ \\ *Universidade Tecnológica Federal do Paraná - UTFPR - COMAT Via do Conhecimento Km 01, \\ 85503-390, Pato Branco - PR, Brazil \\ ${ }^{\dagger}$ Instituto de Física Teórica, UNESP - Universidade Estadual Paulista, Rua Dr. Bento T. Ferraz, \\ 271, Bloco II, 01140-070, São Paulo - SP, Brazil
}

\begin{abstract}
Recently a Minimal and an Ultraminimal Technicolor models were proposed where the presence of TC fermions in other representations than the fundamental one led to viable models without conflict with the known value for the measured S parameter. In this work we apply the results of [5] to compute the masses of the Higgs boson in the case of the Minimal and Ultraminimal Technicolor models.
\end{abstract}

Keywords: Technicolor models, Composite particle models

PACS: $12.60 . \mathrm{Nz}, 12.60 . \mathrm{Rc}$

\section{INTRODUCTION}

The understanding of the gauge electroweak symmetry breaking mechanism is one of the most important problems in particle physics at present. One of the explanations of this mechanism is based on new strong interactions usually named technicolor (TC). Recently a Minimal(MWT) [1] and an Ultraminimal(UMT) [2] TC models were proposed where the presence of TC fermions in other representations than the fundamental one led to viable models without conflict with the known value for the measured $\mathrm{S}$ parameter.

An effective Lagrangian analysis indicates that such models also imply light scalar Higgs bosons $[1,2,3]$. This possibility was investigated and confirmed by us through the use of an effective potential for composite operators [4] and through a calculation involving the Bethe-Salpeter equation (BSE) for the scalar state [5]. The BSE approach is a straightforward one, and our purpose in this work is to complement the studies of dynamical symmetry breaking of Refs. $[4,5]$ in the case of the Minimal and Ultraminimal TC models. We apply the results of Ref.[5] to compute the Higgs boson masses in the case of the Minimal and Ultraminimal TC models.

\section{FERMIONIC SCHWINGER-DYSON EQUATIONS FOR THE MWT AND UMT MODELS}

The Minimal TC model is based on a $S U(2)$ gauge group with two adjoint fermions [1]

$$
\begin{gathered}
Q_{L}^{a}=\left(\begin{array}{c}
U^{a} \\
D^{a}
\end{array}\right)_{L}, \quad U_{R}^{a}, \quad D_{R}^{a}, \quad a=1,2,3, \\
\text { CP1296, XI Hadron Physics } \\
\text { edited by M. Nielsen, F. S. Navarra, and M. E. Bracco } \\
\text { (C) } 2010 \text { American Institute of Physics } 978-0-7354-0848-7 / 10 / \$ 30.00
\end{gathered}
$$


where $a$ is the $S U(2)$ adjoint color index and the left-handed fields correspond to three $\left(S U(2)_{L}\right)$ weak doublets. The Ultraminimal TC model is based on a two colors group with two fundamental Dirac flavors $S U(2)_{L} \times U(1)_{Y}$ charges as described in [2]

$$
T_{L}=\left(\begin{array}{c}
U \\
D
\end{array}\right)_{L}, U_{R}, D_{R}
$$

and also two adjoint Weyl fermions indicated by $\lambda^{f}$ with $f=1,2$, which are singlets un$\operatorname{der} S U(2)_{L} \times U(1)_{Y}$. For phenomenological reasons, in Ref.[2], the appropriate anomalous dimension to generate an acceptable chiral symmetry breaking is obtained with the addition of masses for the adjoint fermions.

The near conformal behavior for these models can be obtained looking at the zero of the two-loop $\beta\left(g^{2}\right)$ function, which is given by

$$
\beta(g)=-\beta_{0} \frac{g^{3}}{(4 \pi)^{2}}-\beta_{1} \frac{g^{5}}{(4 \pi)^{4}},
$$

where $\beta_{0}=(4 \pi)^{2} b=\frac{11}{3} C_{2}(G)-\frac{4}{3} T(R) n_{F}(R)$, and

$$
\beta_{1}=\left[\frac{34}{3} C_{2}^{2}(G)-\frac{20}{3} C_{2}(G) T(R) n_{F}-4 C_{2}(R) T(R) n_{F}\right] .
$$

$C_{2}(R) I=T_{R}^{a} T_{R}^{a}, C_{2}(R) d(R)=T(R) d(G), d(R)$ is the dimension of the representation $R$ and $G$ indicates the adjoint representation.

The main difficulty in TC lies in the small knowledge that we have about the chiral symmetry breaking pattern of such strongly interacting theories. In the models that we will discuss here most of the information about the chiral symmetry breaking comes from the use of effective theories [1,2] and the effective potential generated by them [3]. Another way to unravel the symmetry breaking pattern in TC theories is through the effective potential for composite operators as computed recently in Ref.[4]. It is also possible to obtain information on the spectrum of TC theories simply looking at the gap equations and their possible solutions, this is the simplest approach and is the point of view to be followed here using some of the results of Ref.[5]. Of course, all these attempts involve a reasonable uncertainty typical of non-perturbative theories, but the full set of results may be able to corner the main characteristics of the broken TC theory, i.e. masses and couplings.

In order to discuss the Higgs masses, as performed in Ref.[4, 5], we need to know the solution of the fermionic Schwinger-Dyson equations, or gap equation, for the Minimal and Ultraminimal TC models.

The two basic parameters that define the gap equation are: The $\beta$ function coefficients appearing in the coupling constant and the Casimir operators resulting from the fermiongauge boson vertex. The gap equation, for fermions in the representation $R$, can be written as

$$
\Sigma(k)=\frac{3 C_{2}(R)}{16 \pi^{2}}\left[\frac{g^{2}\left(k^{2}\right)}{k^{2}} \int_{0}^{k^{2}} \frac{p^{2} d p^{2} \Sigma\left(p^{2}\right)}{p^{2}+m^{2}}+\int_{k^{2}}^{\infty} \frac{d p^{2} g^{2}\left(p^{2}\right) \Sigma\left(p^{2}\right)}{p^{2}+m^{2}}\right],
$$


where the coupling constant $g^{2}\left(p^{2}\right)$ behaves as

$$
g^{2}\left(p^{2}\right)=\frac{g^{2}\left(m^{2}\right)}{1+b g^{2}\left(m^{2}\right) \ln \left(\frac{p^{2}}{m^{2}}\right)},
$$

and where $m \approx \Lambda_{T C}$ is the dynamical mass scale that is assumed to be equal to the TC scale. The factor $b$ in Eq.(5), is the one that comes out from the full behavior of the $\beta$ function in Eq.(3) (i.e., including all fermionic representations). A quite general solution for Eq.(4) is [6, 4]

$$
\Sigma(p)=m\left(\frac{m^{2}}{p^{2}}\right)^{\alpha}\left[1+b g^{2} \ln \left(\frac{p^{2}}{m^{2}}\right)\right]^{-\gamma(\alpha)},
$$

where $\gamma(\alpha)=\gamma \cos (\alpha \pi)$ and $\gamma=\frac{3 c}{16 \pi^{2} b} . c$ is the quadratic Casimir operator given by

$$
c=\frac{1}{2}\left[C_{2}\left(R_{1}\right)+C_{2}\left(R_{2}\right)-C_{2}\left(R_{3}\right)\right],
$$

and $C_{2}\left(R_{i}\right)$, are the Casimir operators for fermions in the representations $R_{1}$ and $R_{2}$ that form a composite boson in the representation $R_{3}$. If $R_{1}=R_{2}=R$ and $R_{3}$ is the singlet state $c$ is simply reduced to $C_{2}(R)$. The only restriction on this solution is $\gamma>1 / 2$ [7]. This solution can be understood as one ansatz that maps all possible behavior of the gap equation as we vary $\alpha$, since the standard operator product expansion (OPE) behavior for $\Sigma\left(p^{2}\right)$ is obtained when $\alpha \rightarrow 1$, whereas the "extreme walking" TC solution is obtained when $\alpha \rightarrow 0$. As explained at length in Refs.[4, 6] most of the calculations can be performed with the expression of Eq.(6) and afterward we consider the "extreme walking" (or NJL) limit $\alpha=0$. Note that this is the only possible solution that is naturally able to reproduce the top quark mass[6]. In the Minimal TC model we have fermions in the adjoint representation and $R_{1}=R_{2}=G$ in Eq.(7), while in the Ultraminimal TC model the factor $c$, and consequently $\gamma$, have to change in order to consider the Casimir operators of the Dirac fermions in the fundamental representation $\left(c_{F}\right)$ and the Weyl fermions in the adjoint representation $\left(c_{G}\right)$, with condensation occurring in the TC singlet channel.

\section{THE SCALAR BOSONS MASSES IN THE MWT AND UMT MODELS}

In Ref.[5] we obtained the scalar boson mass in the case of an "extreme walking" TC theory through a calculation based on the BSE, which is given by (see Eq.(26) of Ref.[5])

$$
M_{H}^{2(0)} \approx 4 v^{2}\left(\frac{8 \pi^{2} b g^{2}(m)(2 \gamma-1)}{N_{T C} n_{F}}\right)\left(\frac{1}{4} \frac{b g^{2}(m)(2 \gamma-1)}{\left(1+\frac{b g^{2}(m)(2 \gamma-1)}{2}\right)}\right),
$$

where $v \sim 246 \mathrm{GeV}$ is the Standard Model vacuum expectation value (vev) and we are considering a $S U\left(N_{T C}\right)$ group. This equation depends only on the electroweak group 
vev and on the group theoretical factors and number of fermion flavors. Note that Eq.(8) indicates that the scalar masses are lowered in quasi-conformal gauge theories as a consequence of the BSE normalization condition discussed in Ref.[5].

The Higgs mass of the Ultraminimal TC model cannot be obtained directly from the Eq.(8), because in this case we have two scalar composite bosons, that may appear as mixed states formed by fermions in the fundamental and adjoint representations. For instance, the Ultraminimal gap equation has two contributions, one with a Casimir operator for fermions in the fundamental representation and another with a different Casimir operator for fermions in the adjoint representation, while it is the same $\beta$ function that governs the running of the coupling in the two contributions. Moreover, we shall make use of a trick based on the behavior of the Bethe-Salpeter scalar wave function to estimate the magnitude of the scalar boson mass in the Ultraminimal model. We assume the following relation for the scalar wave functions $\chi(p)_{u m}^{F} \approx\left[\frac{\left(c_{F}\right)^{\gamma_{F}}}{\left(c_{G}\right)^{\gamma_{G}}}\right]_{u m} \chi(p)_{m i}$. Our results for the scalar bosons masses in the Minimal and Ultraminimal walking TC models are displayed in Table I. For comparison we also add the mass of an ordinary walking $S U(2)_{T C}$ with 8 Dirac fermions in the fundamental representation.

TABLE 1. Scalar composite masses of the Minimal, Ultraminimal and ordinary walking $S U(2)$ TC model.

\begin{tabular}{rrrr}
\hline & Walking & Minimal & Ultraminimal \\
\hline$M_{H}(\mathrm{GeV})$ & 142 & 414 & 250 \\
\hline
\end{tabular}

\section{CONCLUSIONS}

To conclude, we presented a discussion about the scalar composite masses, in the case of the Minimal and Ultraminimal TC models. To determine the mass generated for the Higgs boson in these models we considered the BSE approach developed in Ref.[5] so that we complement the results obtained in that work.

\section{ACKNOWLEDGMENTS}

This research was partially supported by the Conselho Nacional de Desenvolvimento Científico e Tecnológico (CNPq).

\section{REFERENCES}

1. R. Foadi, M. T. Frandsen, T. A. Ryttov and F. Sannino, Phys. Rev. D 76, 055005 (2007).

2. T. A. Ryttov and F. Sannino, Phys. Rev. D 78, 115010 (2008).

3. M. Jarvinen, C. Kouvaris and F. Sannino, Phys. Rev. D 81, 064027 (2010).

4. A. Doff, A. A. Natale and P. S. Rodrigues da Silva, Phys. Rev. D 77, 075012 (2008).

5. A. Doff, A. A. Natale and P. S. Rodrigues da Silva, Phys. Rev. D 80, 055005 (2009); A. Doff and A. A. Natale, Phys. Lett. B 677, 301 (2009).

6. A. Doff and A. A. Natale, Phys. Lett. B 537, 275 (2002); Phys. Rev. D 68, 077702 (2003).

7. K. Lane, Phys. Rev. D 10, 2605 (1974). 
Copyright of AIP Conference Proceedings is the property of American Institute of Physics and its content may not be copied or emailed to multiple sites or posted to a listserv without the copyright holder's express written permission. However, users may print, download, or email articles for individual use. 